\title{
Lattice Models of Disorder with Order
}

\author{
Alberto Petri \\ CNR, Istituto “O.M. Corbino”, via del Fosso del Cavaliere 100, 00133 Roma, Italy \\ Unità INFM, Università La Sapienza, p.le Aldo Moro 2, 00185 Roma, Italy
}

Received on 17 April, 2003

\begin{abstract}
This paper describes the use of simple lattice models for studying the properties of structurally disordered systems like glasses and granulates. The models considered have crystalline states as ground states, finite connectivity, and are not subject to constrained evolution rules. After a short review of some of these models, the paper discusses how two particularly simple kinds of models, the Potts model and the exclusion models, evolve after a quench at low temperature to glassy states rather than to crystalline states.
\end{abstract}

\section{Introduction}

In recent years, there is growing interest in systems commonly found in disordered structural configurations such as glasses and dense granulates. Clearly different in many respects, these two classes of materials share in common the property of displaying stable states which are very far from what would be expected from equilibrium considerations $[1,2]$. As a consequence of this property other features emerge, like slow relaxation and response functions.

Models of disorder are generally based on the presence of quenched, or a priori, disorder which takes the system far from ordered configurations. Only recently it has been observed that lattice models capable of ordering can also well reproduce many properties of disordered systems. On the other hand glass formers in nature usually have crystalline states as ground states, but this does not prevent them from being frequently found in some glassy state. The situation for a granular system is in general different, insofar as irregular grains inhibit the evolution of ordered states. Nevertheless even in the case of identical beads it is practically impossible to arrange them into an ordered fashion merely by supplying the energy to them.

The aim of this paper is to briefly review some lattice models employed in the description of the slow dynamics of glasses and dense granular matter, which do not possess any a priori disorder nor long range interactions. We shall not discuss in detail the models, but just recall some of the main results, addressing the interested reader to the bibliography. More attention will be drawn to some recent observations regarding the condition for the emergence of glassy phases in some models of this kind.

The paper is structured as follows: in Section II exclusion models and hard particle models are briefly introduced together with their employment in irreversible dynamics; Section III is devoted to their use in the description of dense granular matter and related results; Section IV describes spin models which have been recently shown to exhibit glassy states and associated slow dynamics; finally in Sec. V some new ideas are illustrated on when and why glassy states are generated in the place of crystalline states in some lattice models.

\section{Exclusion and hard particle models}

Flory [3] modeled the irreversible deposition of dimers on a one-dimensional surface by the random sequential adsorption (RSA) of particles on a lattice. In this model, particles are placed one at time at randomly chosen positions of a linear chain of sites ("hard particles"). Each particle occupies two lattice sites and cannot overlap other particles. Flory was able to show that, despite its stochastic nature, this process leads, in the limit of an infinite lattice, to a well defined percentage of covering which he computed exactly. Soon after the same model was extended to two-dimensional systems and to a variety of particles, also including desorption and cooperative processes (see $[4,5]$ for review). The continuous version of RSA is sometimes called the car parking problem.

Diffusivity, which accounts for particle rearrangement and relaxation, was also introduced in RSA models giving rise to the so called RSAD models (see $[6,7]$ ). In addition, multilayer deposition has been investigated [7]. RSAD multilayer models are the base of the most common lattice models for dense granular materials, as will be explained below.

Exclusion models represent a slight modification of hard particle models. In the latter each particle occupies a certain set of lattice sites that cannot be occupied by other particles. In exclusion models, each particle occupies one single site, and excludes a certain neighbour set from being occupied by other particles. Depending on the exclusion rules, a one-toone correspondence can sometimes be drawn between the two kinds of models. 
Exclusion models have been extensively investigated in relation to equilibrium phase transitions [8]. There identical particles are randomly deposited or retired from a $d$ dimensional lattice at a rate determined by the value of a chemical potential; in addition particles can diffuse along the lattice (in accordance with the exclusion rules), allowing the system to look for configurations of the maximum density compatible with the imposed value of the chemical potential $\mu$. When $\mu$ is taken to infinity the maximum allowed density in reached with particles arranged in an ordered phase. A remarkable result of these models is that they are able to describe both first and second order phase transitions as a function of the space dimensionality and of the number of excluded sites [8]. On the contrary, when the chemical potential in RSAD processes is changed abruptly, diffusion is not always sufficient to drive the system into an ordered phase. When this happens the ordering process is generally very slow, with density evolving algebraically in time.

\section{Models for granulars}

A process widely investigated in the physics of granular materials is the compaction, that is the decrease of the volume fraction (the ratio between the total volume of the constituent grains and the volume macroscopically occupied by the system) of a spatially limited system, subject to gravity, under the action of an external perturbation. The energy supplied by the perturbation (e.g. a mechanical perturbation like tapping or shaking) must be comparable with the variation in potential energy required to rearrange the system structure. The process exhibits rather a rich behaviour with reversible and irreversible cycles as a function of the perturbation amplitude [9], and logarithmic [10] or stretched exponential laws [11] in the number of tapping.

Lattice models with only the excluded volume constraint have proven to be good models for dense granular systems. They are all essentially based on multilayer sequential deposition [12], and have been so far investigated in $1+1$ dimensions. The lattice is considered to lay in the vertical position. It is initially prepared by sequentially dropping extended hard particles from the top. Each particle proceeds downwards until it hits the bottom of the lattice or a previously deposited particle. Excluded volume effects prevent the process from generating ordered configurations and an initial state with many defects and low volume fraction $\rho$ is usually obtained. Then an external mechanical perturbation is activated by turning on upwards diffusion along the lattice meshes for a finite time (the duration of the tapping) and then allowing only downwards and horizontal diffusion in order to mimic the relaxation process under the effect of the gravity field. By periodically turning upwards diffusivity on and off the effect of multiple tapping cycles can be investigated, modulating their intensity by changing the ratio between upwards and downwards-horizontal diffusion.

The so called "Tetris" model introduced by Caglioti et al. [13] in its original version is based on dimers placed on a lattice with meshes tilted of $45^{\circ}$ and, when tapped, shows a logarithmic increase of the volume fraction, in agreement with [9]. More generally, "T" shaped particles have also been considered, showing that the model possesses a thermodynamic-like temperature which is numerically measurable through the fluctuation-dissipation relations [14] and which coincides with a configurational pseudo-temperature proposed for granular matter [15]. The latter has also been measured in a system of monodisperse hard spheres on lattice [16].

Dimers placed on lattice with non tilted meshes also exhibit very well defined scaling laws [17] but with stretched exponential (the so called Kohlrausch-Williams-Watts law) rather than logarithmic compaction:

$$
\rho(t)=\rho_{\infty}-\Delta \rho \exp \left(-t / \tau_{0}\right)^{\beta} .
$$

A similar behaviour has been found in recent extensive experiments [11] and in a generalization of the model which includes friction [18].

\section{Models for glasses}

Lattice gas models with constrained dynamics [19] exhibit many of the properties of glass dynamics, such as slow relaxation, two step relaxation, vanishing diffusivity, ageing, etc. Other kinds of models with constrained dynamics have also been introduced and investigated yielding similar results [20-24].

A class of lattice models exhibiting glassy behaviour with neither quenched disorder nor constrained dynamics are the spin models with many spin interaction whose energy can be written as:

$$
E=-\sum_{<i, j, \ldots, k>} s_{i} s_{j} \ldots s_{k},
$$

where the sum is extended to a finite number of Ising spins: $s_{i}= \pm 1$. A single spin flip Monte Carlo algorithm is generally used for numerical simulation of the dynamics. The four spin model [25] exhibits a discontinuous equilibrium transition in three dimensions, but if quenched quickly enough below the transition temperature, it enters a very long living metastable state with many of the features of glassy systems $[26,27]$. More recently [28] the existence of a dynamic glass transition has been proved for a three spin model on a Bethe lattice that also has a first order transition to a ferromagnetic phase.

Models with geometrical frustration and only two body interaction have been employed as coarse grained approximation of hard sphere models in the continuum limit. A dynamical glass transition has been observed in threedimensional models with a constraint on the local equilibrium density [29] together with a first order transition. Similar results have also been found in [30] and in [31] although in the latter model the crystalline phase does not seem accessible by numerical simulation even at very low cooling rate. 
Some kind of glassy states can also be observed in two dimensional exclusion models. It has been found by numerical simulations [32] that a monolayer RSAD of particles with the exclusion of the first three shells of neighbours ( $N_{3}$ model) is not able to generate a periodic arrangement. The dynamics leads asymptotically to states in which many locally ordered domains of particles are formed, while at the domain boundaries many sites remain empty. Analogous behaviour has been observed in the RSAD of dimers $[33,34]$, whereas in models with exclusion of only two shells of neighbours $\left(N_{2}\right.$ model $)$ the density of empty sites vanishes in the thermodynamic limit [35]. In the next section the properties of these models will be discussed more extensively.

The many spin interaction models, the $N_{3}$ model and the other models referred to above show that glassy states are generated also in models with no quenched disorder or long range interaction and with stable crystalline states. On the other hand, glass formers in general also have crystalline phases; this point deserves consideration and in the next section some ideas on the emergence of glassy vs crystalline states in some lattice models are discussed.

\section{Emergence of glassy states in lattice models}

In this section some properties are reported that have recently emerged [36-39] in the coarsening of the Potts model following a quench and in RSAD dynamics of some exclusion models.

In the Potts model [40] each lattice site can stay in one out of $q$ distinct states, or colours. To each site one associates a variable $\eta_{i}$ that takes the values $1,2, \ldots, q$. Let us write the energy of the $q$ state Potts model as:

$$
U=\sum_{(i j)}\left(1-\delta_{\eta_{i} \eta_{j}}\right)
$$

where $\eta_{i}=1,2, \ldots q$ and the sum is over the nearest neighbour sites. There are $q$ degenerate system ground states with zero energy corresponding to having all sites the same colour.

Starting from an initial disordered configuration a quench of the system can be numerically simulated by letting the system evolve according to the Metropolis dynamics [41] at a temperature $T$ below the temperature at which the model is known to have a phase transition. For $T=0$, large $q$ and periodic boundary conditions, the dynamics leads the system into some frozen state [42] that represents a local minimum in the energy landscape of the finite system with energy higher than the ground state energy. This is not the case for low $q$. In the two-dimensional Ising model, corresponding to $q=2$, the fraction of blocked configurations tends to zero in the thermodynamic limit.

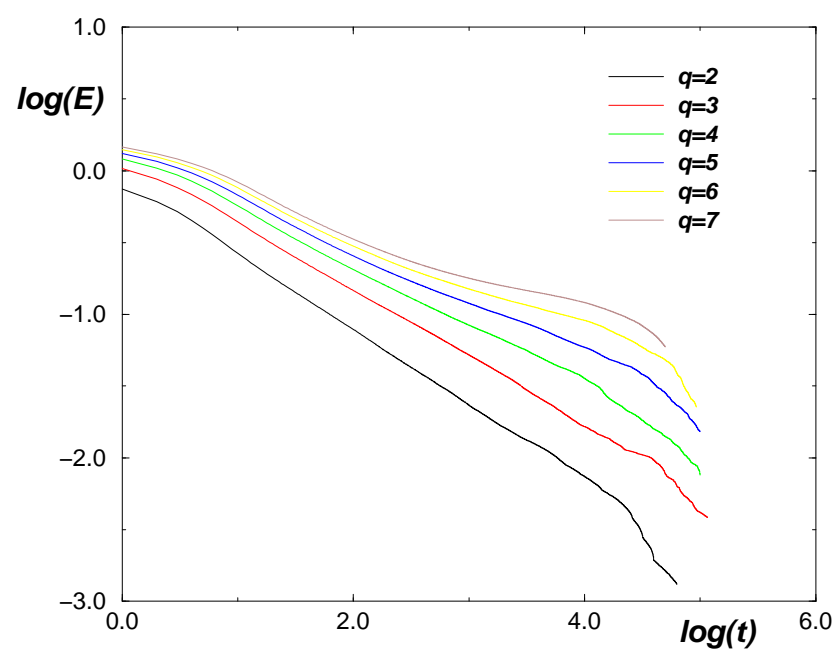

Figure 1. Energy decay in a two dimensional Potts model after a quench at $T=0$. For increasing $q$ larger deviations from $t \propto 1 / \sqrt{(} t)$ are displayed.

A first important consideration concerns such a different behaviour exhibited for different values of $q$. Fig. 1 shows the decrease in time of the energy per site, $E=U / N$, in a system with $N=10^{6}$ sites for some different values of $q$ after a quench at $T=0$. In this case fixed boundary conditions have been used, that is a prefixed colour has been assigned at the beginning to the sites at the system boundary, and cannot change during the simulation. This breaks the symmetry among the different ground states and makes the system converge to the preselected ground state. Since it is expected to follow an algebraic decay of the type $E(t) \propto t^{-\alpha}$ [42, 43], the curves have been plotted on a double logarithmic scale. The figure shows that the lines, which are straight for $q=2$ and $q=3$, decrease their slope and develop a bump as $q$ increases. This suggests at first sight a deviation from the algebraic behaviour. However if the same curves are plotted as functions of $1 / \sqrt{t}$ a linear behavior is observed for all times up to the final relaxation for every value of $q$, showing that the energy decrease in this regime is correctly described by

$$
E(t) \propto t^{-1 / 2}+\text { const }
$$

as shown in Fig. 2 for the case $q=7$. It is the constant term, which depends on $q$, that accounts for the bump and bending observed in log-log scale. After the $1 / \sqrt{t}$ regime the system performs a fast relaxation towards the prefixed ground state.

The second important point is that the time at which the final relaxation occurs increases with lattice size $N$. The same happens for the freezing when periodic boundary conditions are used, but in this case fluctuations of the final energy from sample to sample are larger. Extrapolation of the energy curves leads to conclude that in the thermodynamic limit:

$$
\lim _{t \rightarrow \infty} E(t)=E^{*}(q)
$$

It can be useful to point out that here the thermodynamic limit is taken before the infinite time limit. This is the reason for which the thermodynamical equilibrium state with $E=0$ is not obtained. 


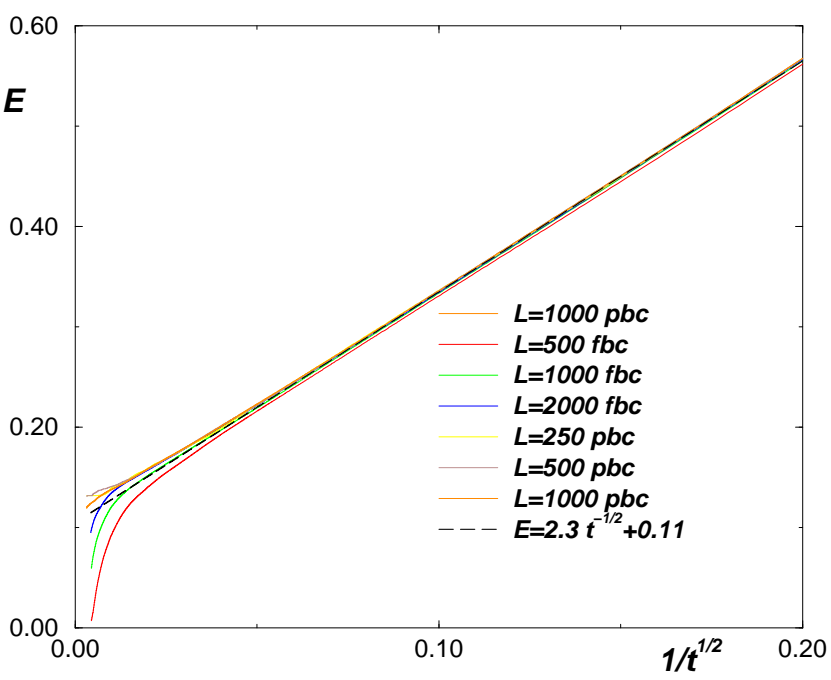

Figure 2. E(t) vs $1 / \sqrt{t}$ for the Potts model with $q=7$ and different size and boundary conditions: $f b c=$ fixed boundary conditions; $p b c=$ periodic boundary conditions. A common regime is shown whose duration increases with the system size.

The residual energy term (5) is due to interfaces separating homogeneous domains with different values of $q$ that do not disappear when $t \rightarrow \infty$. Moreover, since for $q$ large enough this term does not vanish when $N \rightarrow \infty$, it can be concluded that the total length of the interfaces is of order $N$. This property can be used to define the glassy state in this kind of model, and is observed for any $q>4$. For $q<4$ the residual energy vanishes in the thermodynamic limit, showing absence of interfaces (crystalline state for $q=2$ ) or at most a total length of order $\sqrt{N}$ (polycrystalline state for $q=3$ ). The case $q=4$ is critical insofar as relaxation is not $1 / \sqrt{t}$; by including a logarithmic correction $E(t) \propto \sqrt{\ln t} / t+E^{*}$ it is compatible with $E^{*}=0$.

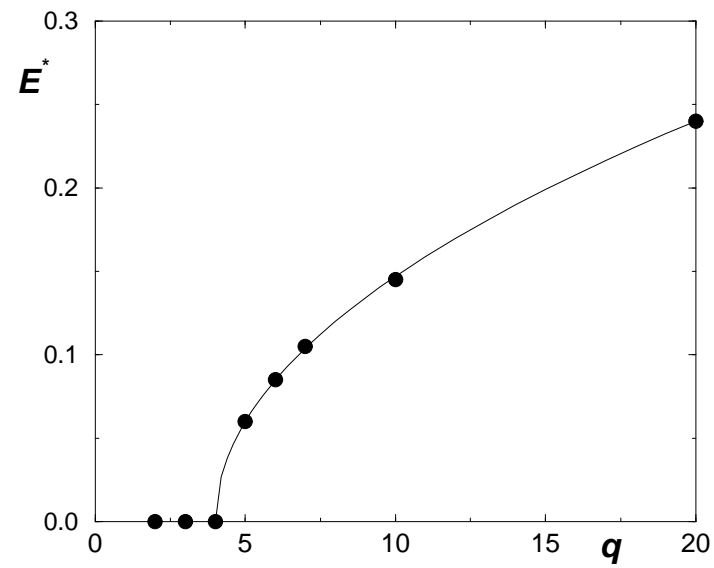

Figure 3. Residual energies in the Potts model after a quench at $T=0$ as a function of the number of states $q$.

Simulations demonstrate that the residual energy $E^{*}(q)$ is a monotonic function of $q$. This is shown for some values of $q$ in Fig. 3 together with the fitting curve $E^{*}(q)=$ $\left(q-q_{c}\right)^{0.5}$ for $q \geq q_{c}=4$. This suggests a relationship between a non zero residual energy and the discontinuous transition in the equilibrium phase diagram of the model dis- played when $q>4$. Such a conjecture is made stronger by the observation of an analogous behaviour in the case of exclusion models. They are predicted to belong to the same universality class as the Potts model [44], with a correspondence between the number $p$ of excluded sites per particle and $q$. In the $N_{1}$ model $p=2$, whereas $p=4$ and $p=5$ in the $N_{2}$ and $N_{3}$ models respectively. In these systems the density of deposited particles plays a role similar to that of energy in the Potts model; in fact one can associate an energy to the density by means of the chemical potential $\mu$. In particular, the density is maximum, and the energy is minimum, in the state with the closest allowed packing of particles, corresponding to a periodic arrangement (ground state). Any deviation from this value signals the presence of residual empty sites (defects). By assuming $E=0$ for the state with the closest packing, an energy $E=\mu \rho_{d}$ corresponds to a density of defects $\rho_{d}$. The equilibrium diagrams of these systems exhibit continuous transition for $p=2$ and $p=4$ and discontinuous transition for $p=5[8,45,44]$. Thus zero density of defects is expected for $p=2$ (crystalline states), a vanishing one for $p=3,4$ (polycrystalline states) and glassy states with a number of defects $O(N)$ for $p=5$. This has indeed been observed [32, 35, 37] and has been confirmed by further numerical simulations to be largely independent on the chosen parameters [38, 39]. On a triangular lattice $N_{1}$ shows an equilibrium first order transition, whereas the transition is discontinuous for the $N_{1}$ and the $N_{2}$ models. Numerical simulations confirm the prediction of crystalline states for $N_{1}$ but are not yet conclusive for $N_{2}$ and $N_{3}$ [38]. It can be interesting to mention that a kind of polycrystalline state, in the sense defined above, has also been observed in the quench of the three-dimensional Ising model [46].

\section{Summary}

The work done in recent years has shown that lattice models with crystalline states and finite connectivity are capable of reproducing many properties of structurally disordered systems like granulates and glasses. This is of relevance for the understanding of the underlying mechanisms, also in view of similar behaviours exhibited by other kinds of models whose features can however be traced back to different origin [47].

The analysis of the conditions under which a lattice model with crystalline states may exhibit glassy states has been recently carried out on simple models such as exclusion and Potts models showing that it is the competition among a number of equivalent ground states that drives the systems away from crystallisation. When this number is small crystallisation or polycrystallisation take place instead. This result is obtained by inverting the usual order of performing the infinite time and the thermodynamic limits, and suggests a relationship between the observed behaviour and the equilibrium properties of the systems, with glassy behaviour associated to the presence of a first order transi- 
tion which is generallly also a feature of the other models described in this paper.

Many thanks are due to T. Tomé and M. J. de Oliveira, with whom most of the work described in Sec. V has been developed, and to F. Dalton for revising the manuscript.

\section{References}

[1] P. G. Debenedetti and F. H. Stillinger, Nature 410, 259 (2001).

[2] H.M. Jaeger, S.R. Nagel and R.P Behringer, Rev. Mod. Phys. 68, 1259 (1996).

[3] P.J. Flory, J. Am. Chem. Soc. 611528 (1939).

[4] J. W. Evans, Rev. Mod. Phys. 65, 1281 (1993).

[5] J. W. Evans, in Nonequilibrium Satistical Mechanics in One Dimension, edited by V. Privman (Cambridge University Press, Cambridge, 1997), p. 205.

[6] M. C. Bartelt and V. Privman, J. Mod. Phys. B 5, 2883 (1991).

[7] P. Nielaba, in Nonequilibrium Satistical Mechanics in One Dimension, edited by V. Privman (Cambridge University Press, Cambridge, 1997), p. 229.

[8] L. K. Runnels, in Phase Transitions and Critical Phenomena, edited by C. Domb and M. S. Green, Vol. 2, (Academic Press, London, 1972), p. 305.

[9] E. R. Nowak, J. B. Knight, E. Ben-Naim, H. M. Jeaeger and S. R. Nagel, Phys, Rev. E 57, 1971 (1998).

[10] J. B. Knight, C. G. Fandrich, C. N. Lau, H. M. Jeaeger and S. R. Nagel, Phys, Rev. E 51, 3957 (1995).

[11] P. Philippe and D. Bideau, Europhys. Lett. 60, 677 (2002).

[12] M. J. de Oliveira and A. Petri, J. Phys. A 31, L425 (1998).

[13] E. Caglioti, V. Loreto, H. J. Herrmann and M. Nicodemi, Phys. Rev. Lett. 79, 1575 (1997).

[14] A. Barrat, V. Colizza and V. Loreto, Phys. Rev. E 66, 111310 (2002).

[15] S. F. Edwards in Granular Matter: An Interdisciplinary Approach edited by A. Mehta, (Springer, New York, 1994), p.121.

[16] A. Fierro, M. Nicodemi and A. Coniiglio, Europhys. Lett. 59, 642 (2002).

[17] C. Fusco, P. Gallo, A. Petri and M. Rovere, Phys. Rev. E 65, 26127 (2002).

[18] C. Fusco, A. Fasolino, P. Gallo, A. Petri and M. Rovere, Phys. Rev. E 66, 31301 (2002).

[19] W. Kob and H. C. Andersen, Phys. Rev. E 48, 4364 (1993).

[20] L. Peliti and M. Sellitto, J. Phys. IV (France) 8, 49 (1998).

[21] G. H. Fredrickson and H. C. Andersen, Phys. Rev. Lett. 53, 1244 (1984).
[22] M.E.J. Newman and C. Moore, Phys. Rev. E 60, 5068 (1999).

[23] A. Buhot and J. P. Garrahan Phys. Rev. E 64, 021505 (2001) and references therein.

[24] L. Davison, D. Sherrington, J. P. Garrahan and A. Buhot, J. Phys. A 34, 5147 (2001).

[25] A. Lipowski, J. Phys. A 30, 7365 (1997).

[26] A. Lipowski and D. Johnston, J. Phys. A 33, 4451 (2000).

[27] M. R. Swift, H. Bokil, R. D. M. Travasso and A. J. Bray, Phys. Rev. B 62, 11494 (2000).

[28] S. Franz, M. Mezard, F. Ricci-Tersenghi, M. Weigt, R. Zecchina, Europhys. Lett. 55, 465 (2001).

[29] G. Biroli and M. Mezard, Phys. Rev. Lett. 88, 025501 (2002).

[30] M. Weigt, A. K. Hartmann, cond-mat/0210054.

[31] M. Pica Ciamarra, M. Tarzia, A. de Candia and A. Coniglio cond-mat/0210144.

[32] E. Eisenberg and A. Baram, J. Phys. A 33, 1729 (2000).

[33] S. A. Grigera, T. S. Grigera and J. R. Grigera, Phys. Lett. A 226, 124 (1997).

[34] C. Fusco, P. Gallo, A. Petri and M. Rovere, J. Chem. Phys. 114, 7563 (2001).

[35] E. Eisenberg and A. Baram, Europhys. Lett. 44, 168 (1998).

[36] M. J. de Oliveira and A. Petri, Phil. Mag. B 82, 617 (2002).

[37] J. S. Wang, P. Nielaba and V. Privman, Physica A 199, 527 (1993).

[38] M. J. de Oliveira, A. Petri and T. Tomé, in Modeling of Complex Systems: Seventh Granada Lectures edited by P. L. Garrido and J. Marro, (American Institute of Physics, 2003), p. 139.

[39] M. J. de Oliveira, A. Petri and T. Tomé, submitted for publication.

[40] F. Y. Wu, Rev. Mod. Phys. 54, 235 (1982).

[41] N. Metropolis, A. W. Rosenbluth, M. N. Rosenbluth, A. H. Teller and E. Teller, J. Chem. Phys. 21, 1087 (1953).

[42] M. P. Anderson, G. S. Grest and D. J. Srolovitz, Phil. Mag. B 59, 293 (1989),

[43] B. Derrida, P. M. C. de Oliveira and D. Stauffer, Physica A 224, 604 (1996).

[44] E. Domany, M. Schick and J. S. Walker, Phys. Rev. Lett. 38, 1148 (1977).

[45] J. Orban, J. D. Van Belle, J. Phys. A 15, L501 (1982).

[46] V. Spirin, P. L. Krapivsky, and S. Redner, Phys. Rev. E 65, 016119 (2002).

[47] S. Franz, R. Mulet, G. Parisi, Phys. Rev. E 65, 021506 (2002). 